\title{
Resposta de glyphosate em mucuna-preta desenvolvida em diferentes temperaturas
}

\author{
Glyphosate response in velvet bean developed in different temperatures \\ La respuesta al glifosato en el frijol terciopelo se desarrolló a diferentes temperaturas
}

Recebido: 28/03/2021 | Revisado: 02/04/2021 | Aceito: 09/04/2021 | Publicado: 19/04/2021

\author{
Roque de Carvalho Dias \\ ORCID: https://orcid.org/0000-0001-5433-5373 \\ Universidade Estadual Paulista, Brasil \\ E-mail: roquediasagro@gmail.com \\ Diego Gomes Munhoz \\ ORCID: https://orcid.org/0000-0002-1561-182X \\ Universidade Estadual Paulista, Brasil \\ E-mail: diegomgomes77@gmail.com \\ Leandro Bianchi \\ ORCID: https://orcid.org/0000-0003-4779-9246 \\ Universidade Estadual Paulista, Brasil \\ E-mail: leandro_bianchii@hotmail.com \\ Vitor Muller Anunciato \\ ORCID: https://orcid.org/0000-0003-4349-1834 \\ Universidade Estadual Paulista, Brasil \\ E-mail: vitor.muller@gmail.com \\ Leandro Tropaldi \\ ORCID: https://orcid.org/0000-0003-1592-5148 \\ Universidade Estadual Paulista, Brasil \\ E-mail: 1.tropaldi@unesp.br \\ Paulo Vinicius da Silva \\ ORCID: https://orcid.org/0000-0003-4647-5602 \\ Universidade Federal da Grande Dourados, Brasil \\ E-mail: paulovsilva@ufgd.edu.br \\ Caio Antonio Carbonari \\ ORCID: https://orcid.org/ 0000-0002-0383-2529 \\ Universidade Estadual Paulista, Brasil \\ E-mail: caio.carbonari@unesp.br \\ Edivaldo Domingues Velini \\ ORCID: https://orcid.org/ 0000-0003-0431-5942 \\ Universidade Estadual Paulista, Brasil \\ E-mail: velini@uol.com.br
}

\begin{abstract}
Resumo
A mucuna-preta é uma leguminosa anual, que nos últimos anos assumiu posição de destaque como uma importante planta daninha em várias regiões. O controle químico constitui-se umas das principais ferramentas de manejo de plantas daninhas, contudo, diferentes condições climáticas podem influenciar consideravelmente a eficácia de muitas moléculas. O glyphosate é um dos herbicidas sistêmicos, de amplo espectro de controle, amplamente utilizado para o controle químico de plantas daninhas. Dessa forma, o objetivo desse trabalho foi avaliar as respostas de crescimento de plantas de mucuna-preta em diferentes temperaturas submetidas a doses de glyphosate. Foram realizados dois experimentos, em casa de vegetação, em delineamento inteiramente casualizado, com cinco repetições. Os tratamentos foram constituídos por nove doses de glyphosate $\left(\mathrm{g} \mathrm{ha}^{-1}\right.$ e.a.): 2,$81 ; 5,62 ; 11,25 ; 22,5 ; 45,0 ; 90,0 ; 180,0$; 360,0 e 720,0, além de um tratamento controle (sem aplicação de herbicida). Foram realizadas avaliações visuais de fitointoxicação aos 7, 14, 21 e 28 dias após a aplicação (DAA), e aos 28 DAA massa seca da parte aérea das plantas, além da extração e quantificação da cera epicuticular. Doses de glyphosate inferiores a 22,5 g ha-1 e.a. não ocasionaram nenhum efeito nas plantas e nas doses superiores a $360 \mathrm{~g} \mathrm{ha}^{-1}$ e.a. proporcionou a morte da mucuna-preta em ambos os experimentos. A resposta da mucuna-preta nas doses intermediárias de glyphosate variou e associou-se as diferentes condições de temperatura que provocaram diferenças nas quantidades de ceras nos experimentos realizados. As plantas de mucuna-preta respondem de maneira diferente ao herbicida glyphosate em condições de temperatura diferente.
\end{abstract}

Palavras-chave: Adubo verde; EPSPS; Planta daninha.

\begin{abstract}
Velvet bean is an annual legume, which in recent years has assumed a prominent position as an important weed in several regions. Chemical control is one of the main tools for weed management, however, different climatic conditions can considerably influence the effectiveness of many molecules. Glyphosate is one of the systemic
\end{abstract}


herbicides, with a wide spectrum of control, widely used for chemical control of weeds. Thus, the objective of this work was to evaluate the growth responses of velvet bean plants at different temperatures submitted to glyphosate doses. Two experiments were carried out in a greenhouse, in a completely randomized design, with five replications. The treatments consisted of nine doses of glyphosate $\left(\mathrm{g} \mathrm{ae} \mathrm{ha}^{-1}\right) 2.81 ; 5.62 ; 11.25 ; 22.5 ; 45.0 ; 90.0 ; 180.0 ; 360.0$ and 720.0 , in addition to a control treatment (without herbicide application). Visual phytotoxicity evaluations were performed at 7, 14, 21 and 28 days after application (DAA), and at 28 DAA shoot dry matter of the plants, in addition to extraction and quantification of the epicuticular wax. Doses of glyphosate less than $22.5 \mathrm{~g}$ ae ha ${ }^{-1}$ did not cause any effect on plants and doses greater than $360 \mathrm{~g}^{2}$ e ha-1 caused the death of the velvet bean in both experiments. The response of the velvet bean in the intermediate doses of glyphosate varied and was associated with the different temperature conditions that caused differences in the amounts of waxes in the experiments carried out. Velvet bean plants respond differently to the herbicide glyphosate under different temperature conditions.

Keywords: Green manures; EPSPS; Weed.

\section{Resumen}

El frijol terciopelo es una leguminosa anual, que en los últimos años ha asumido una posición destacada como maleza importante en varias regiones. El control químico es una de las principales herramientas para el manejo de malezas, sin embargo, las diferentes condiciones climáticas pueden influir considerablemente en la efectividad de muchas moléculas. El glyphosate es uno de los herbicidas sistémicos, con un amplio espectro de control, muy utilizado para el control químico de malezas. Así, el objetivo de este trabajo fue evaluar las respuestas de crecimiento de plantas de frijol terciopelo a diferentes temperaturas sometidas a dosis de glyphosate. Se realizaron dos experimentos en invernadero, en un diseño completamente al azar, con cinco repeticiones. Los tratamientos consistieron en nueve dosis de glyphosate $\left(\mathrm{g}\right.$ e.a. $\left.\mathrm{ha}^{-1}\right) 2,81 ; 5,62 ; 11,25 ; 22,5 ; 45,0 ; 90,0 ; 180,0 ; 360.0$ y 720.0, además de un tratamiento control ( in aplicación de herbicida). Se realizaron evaluaciones de fitotoxicidad visual a los 7, 14, 21 y 28 días después de la aplicación (DAA), y a los 28 DAA brote la materia seca de las plantas, además de la extracción y cuantificación de la cera epicuticular. Dosis de glyphosate menores de 22,5 g e.a. ha $^{-1}$ no causaron ningún efecto en las plantas y dosis mayores de $360 \mathrm{~g}$ e.a. ha ${ }^{-1}$ provocaron la muerte del frijol terciopelo en ambos experimentos. La respuesta del frijol terciopelo a las dosis intermedias de glyphosate varió y se asoció con las diferentes condiciones de temperatura que provocaron diferencias en las cantidades de ceras en los experimentos realizados. Las plantas de frijol terciopelo responden de manera diferente al herbicida glyphosate en diferentes condiciones de temperatura.

Palabras clave: Abonos verdes; EPSPS; Maleza.

\section{Introdução}

A mucuna-preta (Mucuna pruriens var. utilis (Wall. ex Wight) Baker ex Burck) é uma leguminosa herbácea anual da família Fabaceae, com ramos trepadores e vigorosos que adapta-se bem a climas tropicais e subtropicais (Wutke, 1993). Muito utilizada como adubo verde devidos aos diversos benefícios, como a fixação de nitrogênio simbiótico, reciclagem de nutrientes, controle de erosão e melhoria do solo em sua estrutura física (Wutke, 1993), supressão de nematoides (Giraldeli et al., 2017) e de plantas daninhas (Silva et al., 2013).

Contudo, devido ao manejo inadequado com a incorporação dessa espécie ao solo com sementes próximas à maturação e aos fatores relacionados às características da semente, como alto vigor, dormência e germinação escalonada, contribuíram para que essa espécie se tornasse uma importante planta daninha no cenário agrícola (Silva et al., 2012).

A mucuna-preta, tal como ocorre com as espécies de corda-de-viola (trepadeiras), se entrelaça da cultura hospedeira prejudicando a absorção da luz ao atingir o ápice das plantas, e consequentemente, prejudique a fotossíntese e a alocação de fotoassimilados. Além disso, em culturas como a cana-de-açúcar, por exemplo, causa prejuízo ao processo de colheita, por dificultar a colheita comprometendo o rendimento operacional da colhedora e a qualidade do produto colhido (Bressanin et al., 2015). Dessa forma, o conhecimento da suscetibilidade associada à necessidade de controle dessa espécie é fundamental frente a sua crescente importância.

O glyphosate tem sido um dos herbicidas mais estudados pela comunidade científica nos últimos quinze anos (Duke, 2018). Em plantas, atua na rota do ácido chiquímico inibindo a atividade da enzima 5-enolpiruvilchiquimato-3-fosfato sintase (EPSPs), que resulta na inibição da biossíntese e redução da disponibilidade dos aminoácidos aromáticos fenilalanina, tirosina e triptofano. É um herbicida de amplo espectro de controle, não seletivo, exceto para as culturas geneticamente modificadas, recomendado para aplicações em pós-emergência de plantas daninhas em culturas anuais e perenes (Duke \& Powles, 2008). 
Vale destacar ainda que nem sempre é possível controlar totalmente as plantas daninhas por meio da aplicação em área total, sendo necessária a utilização de outras medidas, como o caso da "catação química dirigida" ou "repasse" utilizando de máquinas costais ou tratorizadas. Segundo Melo et al. (2013), esse método de controle é comumente utilizado em cana-deaçúcar em espécies de difícil controle, perenizadas ou que ocorrem de maneira escalonada, como o caso da mucuna-preta. Assim, o herbicida glyphosate poderia ser uma alternativa interessante para essa modalidade.

A eficácia dos herbicidas pode ser influenciada por diversos fatores, como as características físico-químicas e dose do herbicida, a espécie a ser controlada (características estruturais próprias), o estádio de desenvolvimento e a biologia da planta daninha, as técnicas de aplicação e os fatores ambientais no momento e após a aplicação dos herbicidas (Cieslik et al., 2017). Entre eles, os fatores climáticos destacam-se a temperatura do ambiente, assim, plantas que respondem a essas variações podem estar sujeitas às influências relacionadas ao desempenho do herbicida e/ou interação entre planta e herbicida (Continero et al., 2019; Trezzi et al., 2021).

O glyphosate é o herbicida mais utilizado em larga escala no mundo inteiro nos últimos anos, porém ainda existem informações limitadas quanto aos efeitos em plantas de mucuna-preta. Dessa forma, entender o efeito do glyphosate em plantas cultivadas em condições distintas é de grande importância. Diante do exposto, o trabalho teve por objetivo avaliar os efeitos de doses de glyphosate em plantas de mucuna-preta.

\section{Metodologia}

Foram realizados dois experimentos (março/abril e julho/agosto de 2019) em casa de vegetação, em delineamento em blocos ao acaso, com cinco repetições. As unidades experimentais foram constituídas por vasos de 1,8 L preenchidos com substrato organomineral, contendo turfa de sphagno, vermiculita expandida, casca de arroz torrefado, calcário dolomítico, gesso agrícola e traços de NPK; condutividade elétrica $(\mathrm{CE})=0,7 \pm 0,3 \mathrm{mS} / \mathrm{cm} ; \mathrm{pH}$ em água ou $\mathrm{KCl}$ 5,5 $\pm 0,5$; densidade das partículas $155 \mathrm{~kg} / \mathrm{m}^{3}$; capacidade de retenção de água $55 \%$. A semeadura da mucuna-preta foi realizada com duas sementes por unidade experimental, na profundidade de 1-2 cm e após a emergência, foi realizado o desbaste, mantendo se uma planta por vaso. Durante a condução do experimento os vasos foram irrigados sempre que necessário, visando manter a umidade necessária para o desenvolvimento das plantas. Além disso, as variações de temperaturas máxima e mínima no interior da casa de vegetação durante a realização dos experimentos foram monitoradas por meio de "datalogger" digital, modelo Elitech RC5 (Figura 1).

Aos 23 dias após a emergência, quando as plantas apresentavam a primeira folha verdadeira expandida, foram realizadas as aplicações do herbicida glyphosate (Roundup Original® CS, $360 \mathrm{~g} \mathrm{~L}^{-1}$ e.a., Monsanto) nas doses de 0; 2,81; 5,62; 11,$25 ; 22,5 ; 45,0 ; 90,0 ; 180,0 ; 360,0$ e 720,0 $\mathrm{g} \mathrm{ha}^{-1}$ e.a., utilizando-se um pulverizador instalado em ambiente controlado, provido de uma barra de pulverização com quatro pontas de pulverização XR $110.02 \mathrm{VS}$, espaçadas entre si em 0,5 m e posicionadas a $0,5 \mathrm{~m}$ de altura em relação à superfície das unidades experimentais. $\mathrm{O}$ sistema foi operado com velocidade de deslocamento de $3,6 \mathrm{~km} \mathrm{~h}^{-1}$, com volume de calda de $200 \mathrm{~L} \mathrm{ha}^{-1}$, pressão constante de $3,0 \mathrm{kgf} \mathrm{cm}^{-2}$, pressurizado por ar comprimido.

No momento da aplicação do glyphosate, coletou-se por repetição, quatro folhas totalmente expandidas aleatórias para a quantificação da cera epicuticular, segundo a metodologia de Hamilton (1995). As amostras coletadas foram introduzidas, separadamente por repetição, em béquer com $100 \mathrm{~mL}$ de clorofórmio por 20 segundos, agitando-se levemente. As soluçães obtidas (cera mais clorofórmio) foram filtradas em papel-filtro e em seguida, foram evaporados em banho-maria, para obtenção do resíduo sólido (cera). A quantificação da cera foi expressa pela quantidade de cera por unidade de área foliar (mg $\mathrm{cm}^{-2}$ ) sendo a determinação da área das folhas com auxílio do medidor de área foliar de bancada LICOR®, modelo LI-3100 Area Meter. 
O efeito das doses de glyphosate foi avaliado por meio do nível de fitointoxicação e massa seca da parte aérea (MSPA). Para as avaliações de fitointoxicação ao herbicida glyphosate, ao 0, 7, 14 e 21 dias após a aplicação (DAA), adotouse a escala de notas visuais que varia de 0 a 100 , na qual "0" correspondeu à ausência total de injúrias e "100" à morte total das plantas (SBCPD, 1995). Aos 28 DAA realizou-se a coleta da parte aérea das plantas para a determinação da MSPA, para isso, as mesmas foram acondicionadas em sacos de papel e secas em estufa com circulação forçada de ar de $60^{\circ} \mathrm{C}$ até atingirem massa constante e, posteriormente, pesada em balança analítica.

Os resultados obtidos foram submetidos à análise de variância pelo teste F. Quando o teste F foi significativo, as variáveis de fitointoxicação e a MSPA foram ajustadas a modelos de regressão não lineares do tipo log-logístico (metodologia de curva-dose-resposta), proposto por Streibig (1988):

$$
y=\frac{a}{\left[1+\left(\frac{x}{b}\right)^{c}\right]}
$$

em que: $\mathrm{x}$ é a dose do herbicida; e a, b e c são parâmetros da curva, de modo que a é a diferença entre o ponto máximo e mínimo da curva, b é a dose que proporciona $50 \%$ de resposta da variável e c é a declividade da curva. Sendo os gráficos elaborados pelo Sigmaplot (Systat Software, versão 12.0, San José, CA).

\section{Resultados e Discussão}

Durante a condução dos experimentos vale destacar que houve diferença de temperatura em que as plantas se desenvolveram (Figura 1). Antes da aplicação das doses do herbicida glyphosate a média de temperatura no primeiro experimento foi de $26,32{ }^{\circ} \mathrm{C}$ em relação a $18,58{ }^{\circ} \mathrm{C}$ do segundo experimento, caracterizando assim, diferenças para essa condição.

Figura 1. Dados diários de temperatura $\left({ }^{\circ} \mathrm{C}\right)$ e momentos em que foram realizados o plantio e aplicação das doses do herbicida glyphosate para o primeiro e segundo experimento, respectivamente.

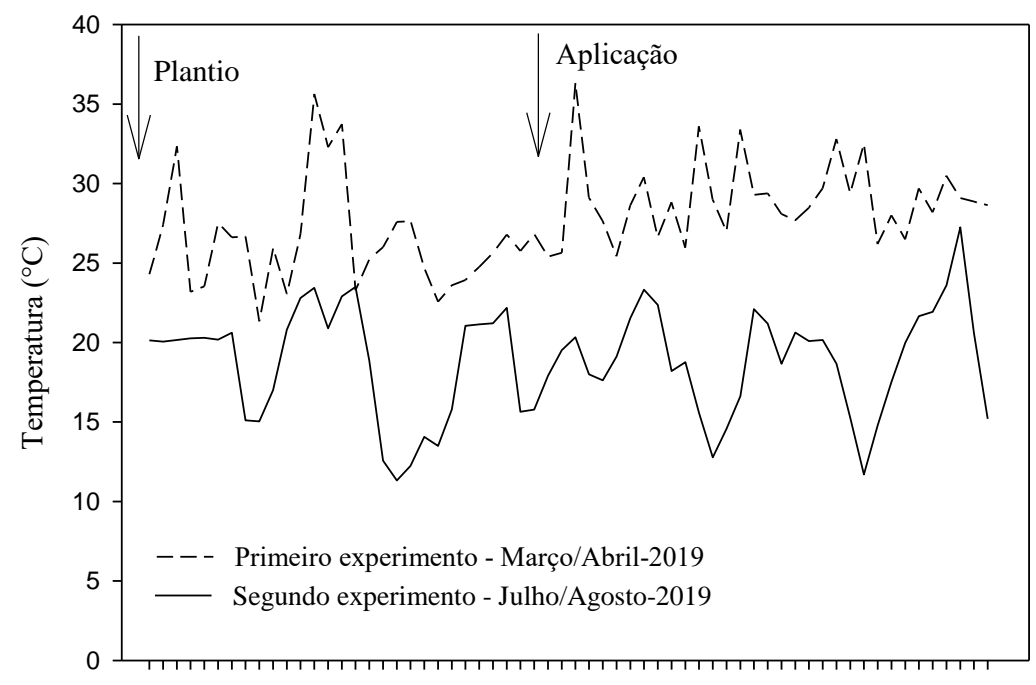

Dias de condução dos experimentos

Fonte: Autores.

Além disso, verificou-se que a quantidade de cera epicuticular extraída (Figura 2) foi maior no primeiro experimento 
$34 \%$ em comparação ao segundo experimento.

Figura 2. Cera epicuticular $\left(\mathrm{mg} \mathrm{cm}^{-2}\right)$ em folhas de mucuna-preta. Médias seguidas pela mesma letra não diferem entre si pelo teste de Tukey $(\mathrm{P}<0,05)$. Barras verticais representam o desvio padrão da média $(\mathrm{n}=5)$.

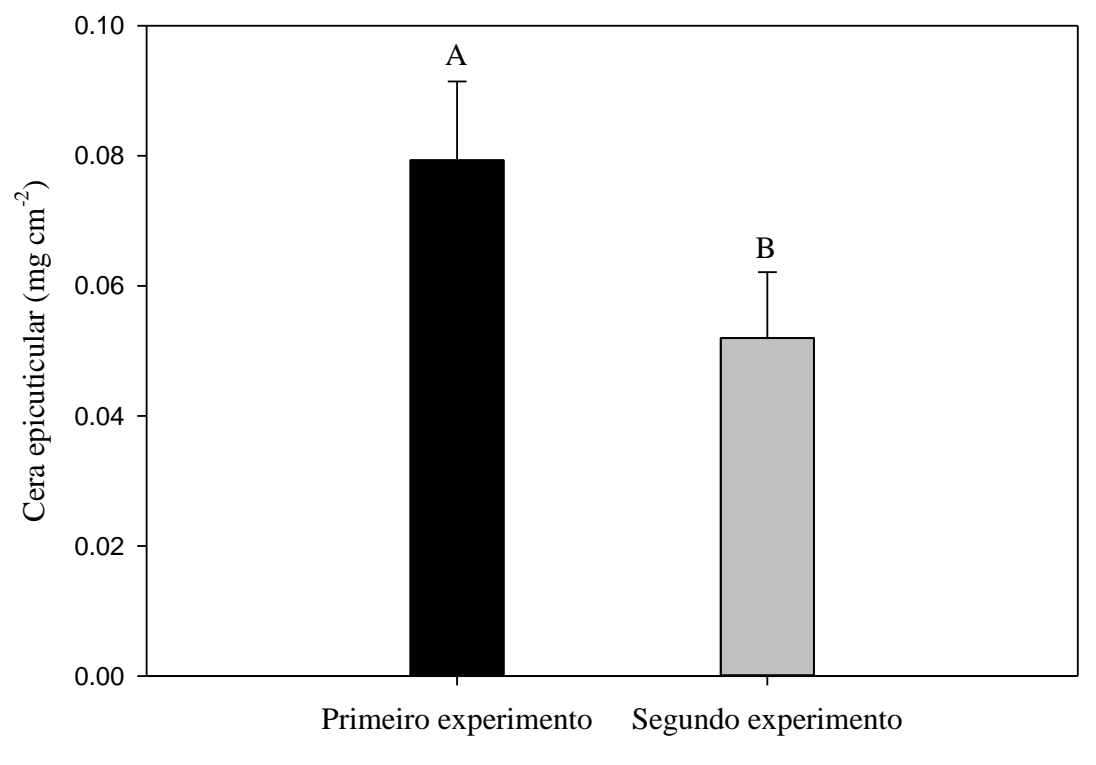

Fonte: Autores.

Nos dois experimentos não foram observados sintomas de fitointoxicação e influência negativa na produção MSPA pelo glyphosate nas doses até 22,5 $\mathrm{g} \mathrm{ha}^{-1}$ e.a. para o primeiro experimento e até $45 \mathrm{~g} \mathrm{ha}^{-1}$ e.a. para o segundo experimento em todos os períodos avaliados (Figuras 3 e 4). Posteriormente, os sintomas se mostraram muito mais intensos no segundo experimento, uma vez que na dose de $180 \mathrm{~g} \mathrm{ha}^{-1}$ e.a. os valores aos 14, 21 e 28 dias após a aplicação (DAA) foram 50\% maiores, no segundo em comparação ao primeiro experimento (Figura 3). 
Figura 3. Porcentagem de fitointoxicação de plantas de mucuna-preta em função das doses de glyphosate (g ha ${ }^{-1}$ e.a.) aos 7 , 14, 21 e 28 DAA. Barras verticais representam o desvio padrão da média $(n=5)$.
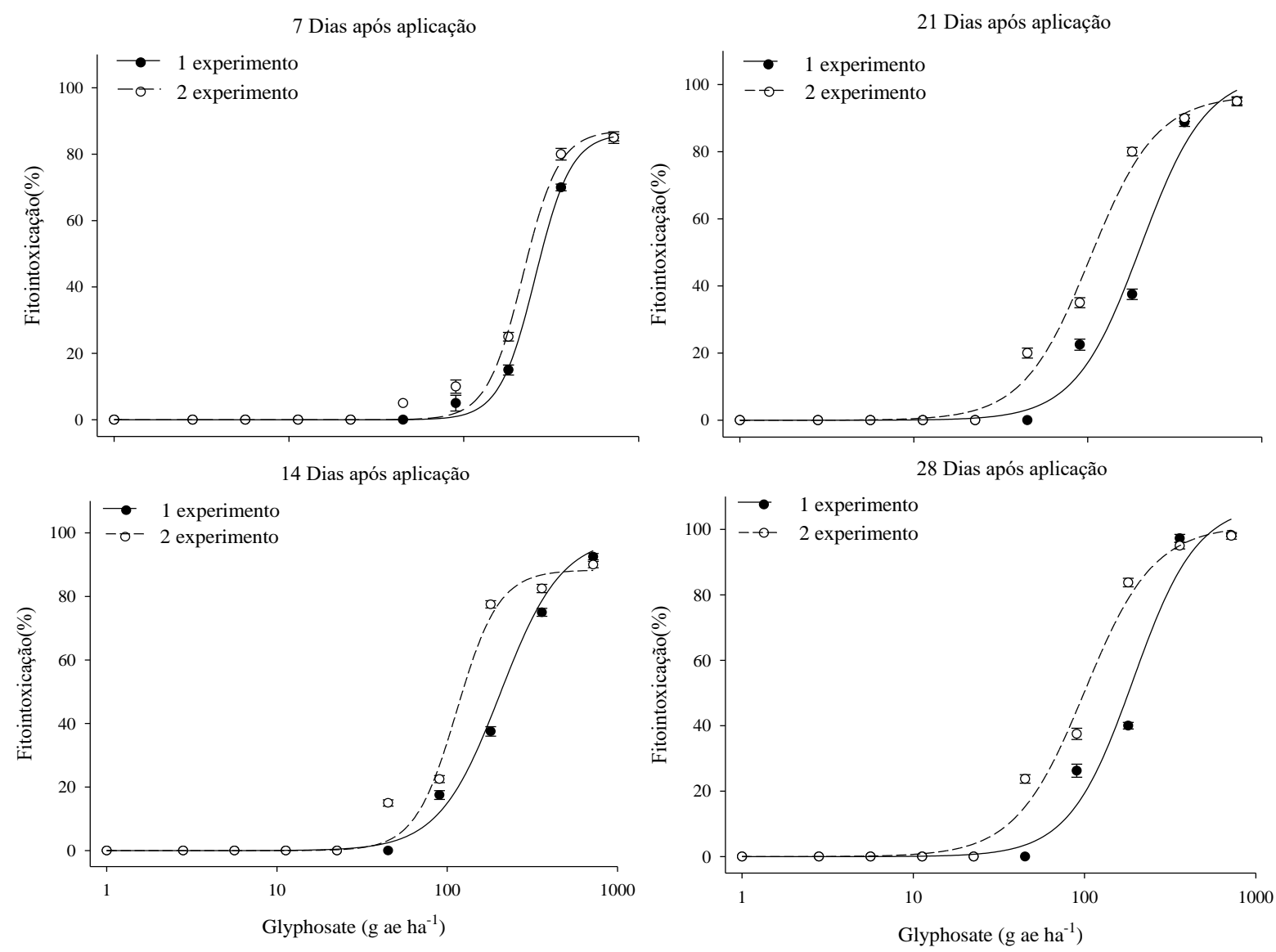

Fonte: Autores.

Refletindo na redução da MSPA aos 28 DAA de cerca de $80 \%$ para $45 \mathrm{~g} \mathrm{ha}^{-1}$ e.a., enquanto que no primeiro experimento esse valor se manteve semelhante ao tratamento controle (sem herbicida) (Figura 4), demonstrando assim, a diferença na resposta desse herbicida as plantas de mucuna-preta em ambos os experimentos. De maneira geral, embora tenha sido verificada diferença na intensidade dos sintomas, estes visualmente foram semelhantes sendo, as folhas de mucuna-preta expostas a doses superiores a $180 \mathrm{~g} \mathrm{ha}^{-1}$ e.a. apresentaram como sintomas principais o amarelecimento das folhas, seguidas de clorose e necrose. 
Figura 4. Massa seca da parte aérea (MSPA) (A) (\%) da parte aérea de plantas de mucuna-preta em função das doses de glyphosate (g ha-1 e.a.) aos 7, 14, 21 e 28 DAA. Barras verticais representam o desvio padrão da média ( $\mathrm{n}=5)$.

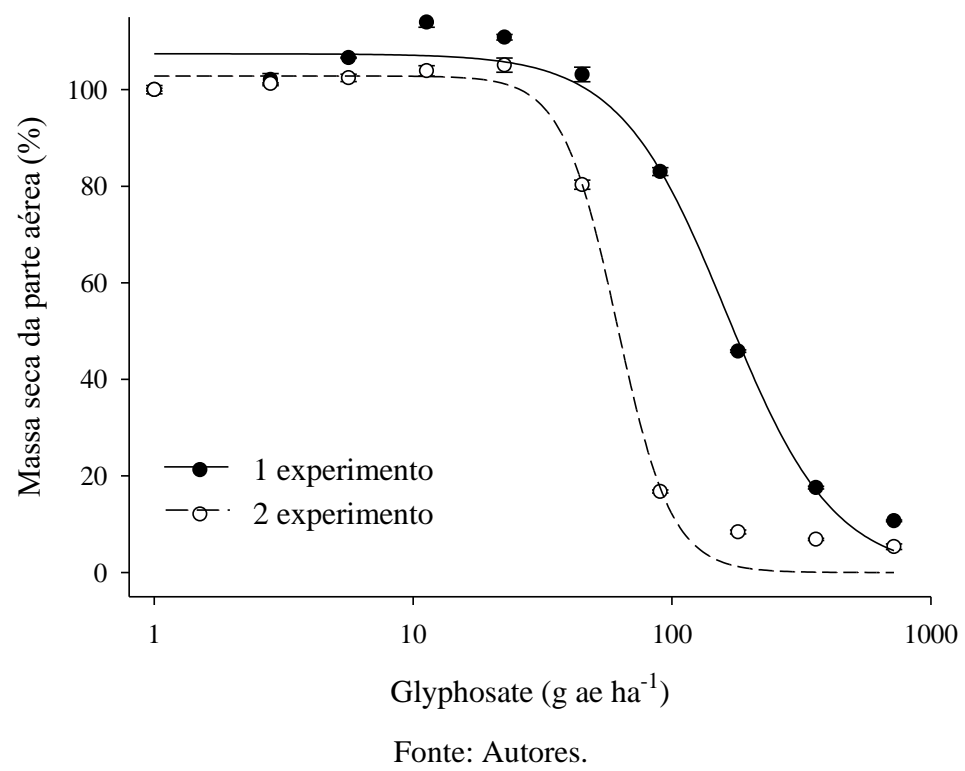

No primeiro experimento aos 7, 14, 21 e 28 DAA, foram necessárias as doses de 256,76, 204,89, 201,51 e 192,10 g ha $^{-1}$ e.a. de glyphosate para o controle $50 \%$ das plantas de mucuna-preta (I50), enquanto que no segundo, as doses foram de 218,16, 114,09, 103,29 e 100,43, respectivamente, demonstrando assim uma maior tolerância das plantas no primeiro experimento (Tabela 1). Da mesma forma, foi requerido em $62 \%$ da dose a mais de glyphosate para a redução em $50 \%$ da MSPA para o primeiro experimento em contrapartida ao segundo (Tabela 2), corroborando com as informações de fitointoxicação citadas anteriormente.

Tabela 1. Estimativas dos parâmetros a, b e c, coeficiente de determinação (r2) do modelo log-logístico em relação fitointoxicação de mucuna-preta submetida a diferentes doses de glyphosate.

\begin{tabular}{|c|c|c|c|c|c|c|}
\hline \multirow{2}{*}{ Variável } & \multirow{2}{*}{ Experimento } & \multirow{2}{*}{$\mathrm{DAA}^{1}$} & \multicolumn{4}{|c|}{ Parâmetros } \\
\hline & & & $\mathrm{a}$ & $\mathrm{b}$ & $\mathrm{c}$ & $\mathrm{r}^{2}$ \\
\hline \multirow{8}{*}{ Fitointoxicação } & \multirow{4}{*}{1} & 7 & 86,26 & 256,76 & $-4,24$ & 0,99 \\
\hline & & 14 & 98,96 & 204,89 & $-2,40$ & 0,99 \\
\hline & & 21 & 103,49 & 201,51 & $-2,30$ & 0,98 \\
\hline & & 28 & 107,91 & 192,10 & $-2,42$ & 0,97 \\
\hline & \multirow{4}{*}{2} & 7 & 87,19 & 218,16 & $-4,21$ & 0,99 \\
\hline & & 14 & 88,32 & 114,09 & $-3,52$ & 0,98 \\
\hline & & 21 & 96,83 & 103,29 & $-2,26$ & 0,99 \\
\hline & & 28 & 101,20 & 100,43 & $-2,15$ & 0,98 \\
\hline
\end{tabular}

${ }^{1}$ DAA: Dias após a aplicação. Fonte: Autores. 
Tabela 2. Estimativas dos parâmetros a, b e c, coeficiente de determinação $\left(\mathrm{r}^{2}\right)$ do modelo $\log$-logístico em relação a massa seca da parte aérea (MSPA) (\%) da parte aérea de mucuna-preta submetida a diferentes doses de glyphosate.

\begin{tabular}{cccccccc}
\hline \multirow{2}{*}{ Variável } & \multirow{2}{*}{ Experimento } & \multirow{2}{*}{ DAA $^{1}$} & \multicolumn{5}{c}{ Parâmetros } \\
\cline { 4 - 8 } & & & $\mathrm{a}$ & $\mathrm{b}$ & $\mathrm{c}$ & $\mathrm{r}^{2}$ \\
\hline \multirow{2}{*}{ Biomassa seca } & 1 & 28 & 107,42 & 162,50 & 2,09 & 0,99 \\
\cline { 2 - 8 } & 2 & 28 & 102,82 & 61,41 & 4,12 & 0,99 \\
\hline
\end{tabular}

${ }^{1}$ DAA: Dias após a aplicação. Fonte: Autores.

Diferenças de temperatura durante a condução dos experimentos, principalmente caracterizada pela temperatura mais elevada no primeiro experimento pode ter influenciado as diferenças na sensibilidade das plantas a aplicação de glyphosate. Essa condição de temperatura elevada pode ocasionar níveis de estresse nas plantas, fazendo com que estas respondem de maneira a evitar a perda de água por transpiração. Como resposta, as plantas fecham os estômatos e produzem mais cera epicuticular, a qual, sendo uma importante barreira à perda de água (Taiz \& Zeiger, 2013), corroborando com os resultados encontrados nesse trabalho. A maior espessura da epiderme pode limitar a penetração e translocação de herbicidas aplicados sobre as folhas conferindo maior tolerância as plantas (Costa et al., 2010). De acordo com Monquero et al. (2004) a tolerância ao glyphosate pode ser explicado devido à dificuldade da penetração dessa molécula. Assim, a quantidade de absorção foliar de herbicidas e, por seguinte a sua eficácia de controle estão diretamente relacionadas aos tipos de estruturas encontradas na folha e à permeabilidade das cutículas que pode variar de acordo com a sua constituição e polaridade dos componentes da cutícula.

Segundo Zanatta et al. (2007), essa alta produção de cera tem sido considerada como uma importante barreira à penetração de herbicidas hidrofílicos. Esses mesmos autores verificaram redução na eficácia de controle de Euphorbia heterophylla L. pelo herbicida glyphosate em condições de estresse ambiental (temperatura e umidade). Para essa mesma espécie, Ferreira et al. (2003) consideraram como principais barreiras foliares à penetração de agroquímicos o alto teor de cera epicuticular, a elevada densidade de laticíferos e a grande espessura da cutícula da face adaxial. A redução na absorção de glyphosate por plantas por estresses abióticos tem sido atribuída à menor hidratação da cutícula (Mcallister \& Haderlie, 1985). No caso dos herbicidas hidrofílicos (baixo Kow, como o glyphosate), a água de hidratação da cutícula e os filamentos de pectina dispersos na matriz da cutina (Velini et al., 2009) são as principais rotas de penetração desse herbicida, assim devido a essa condição, a retenção e a absorção da molécula de glyphosate foi prejudicada em plantas de mucuna-preta. Segundo Delgado et al. (2012) a mucuna-preta (M. pruriens) contém grande quantidade de cera epicuticular em formatos não uniformes e que prejudicam o contato com o glyphosate limitando sua ação.

Já nas maiores doses 360 e $720 \mathrm{~g} \mathrm{ha}^{-1}$ e.a. os valores próximos a 100\% de fitointoxicação foram verificados em ambos os experimentos. Oliveira Neto et al. (2011), estudaram que o glyphosate (1440 $\left.\mathrm{g} \mathrm{ha}^{-1}\right)$ em misturas em tanque com 2,4-D, carfentrazone-ethyl e MSMA tiveram controle de 100\% aos 28 DAA em plantas de mucuna-preta. Em espécies da mesma família da mucuna-preta (Fabaceae) como mata-pasto (Senna obtusifolia (L.) H.S. Irwin \& Barneby) a aplicação de 420 g ha-1 (Barnes \& Oliver, 2003) e $1080 \mathrm{~g} \mathrm{ha}^{-1}$ (Souza et al., 2014) de glyphosate provocou sintomas de fitointoxicação superiores a $80 \%$ aos 7 e 14 DAA, respectivamente. Já ao avaliar a seletividade de espécies nativas como a acácia-monjolo (Acacia

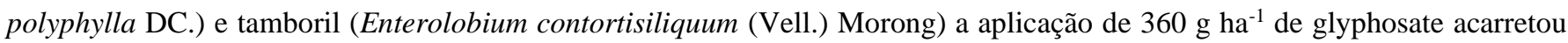
em 60 e $80 \%$ de fitointoxicação, respectivamente, demonstrando alta sensibilidade ao herbicida glyphosate para espécies dessa família (Monquero et al., 2011). Segundo Bressain et al. (2015), a mucuna-preta é uma importante planta daninha no cenário sucroalcooleiro, competindo com as plantas por água, luz e nutrientes, além de prejuízos operacionais na colheita, logo, os resultados obtidos nesse manuscrito representam uma opção de controle dessa espécie de planta daninha.

Dessa maneira, embora existam poucas informações referentes a efeitos abióticos nos efeitos do herbicida glyphosate 
em mucuna-preta, o presente estudo contribui na elucidação dos impactos nas diferenças ambientais no controle de plantas daninhas com herbicidas.

\section{Conclusão}

$\mathrm{O}$ glyphosate em doses superiores a $360 \mathrm{~g} \mathrm{ha}^{-1}$ controlam as plantas de mucuna-preta enquanto que doses inferiores a 22,5 $\mathrm{g} \mathrm{ha}^{-1}$ e.a. não há efeito nas plantas. Variações ambientais como a temperatura influenciam a resposta do glyphosate a mucuna-preta.

\section{Agradecimentos}

O presente trabalho foi realizado com apoio da Coordenação de Aperfeiçoamento de Pessoal de Nível Superior Brasil (CAPES) - Código de Financiamento 001. Ao Conselho Nacional de Pesquisa (CNPq) pelo apoio financeiro a pesquisa através do Projeto (Processo n. 140683/2018-6).

\section{Referências}

Barnes, J. W. \& Oliver, L. R. (2003) Cultural practices and glyphosate applications for sicklepod (Senna obtusifolia) control in soybean (Glycine max). Weed Technology, 17(3), 429-40.

Bressanin, F. N., Giancotti, P. R. F., Neto, N. J., do Amaral, C. L. \& Alves, P. L. D. C. A. (2015) Eficácia de herbicidas aplicados isolados em pré e pósemergência no controle de mucuna-preta. Revista Brasileira de Ciências Agrárias, 10(3), 426-31.

Cieslik, L. F., Vidal, R. A., Machado, A. B. \& Trezzi, M. M. (2017). Eficácia do fluazifop-p-butil em função do tempo de aplicação e da dose do herbicida. Planta Daninha, 35.e017167349.

Contiero, R. L., Rios, F. A., Biffe, D. F., Braz, G. B., Constantin, J., de Oliveira Jr, R. S., Gheno, E. A. \& Lucio, F. R. (2019). Effect of day time climatic conditions associated with different 2,4-D formulations on spray deposition and weed control. Journal of Environmental Science and Health, Part B, 54(10), 803-09.

Costa, N. V., Martins, D., Rodella, R. A., Rodrigues, A. C. P. \& Cardoso, L. A. (2010). Efeito do trinexapac-ethyl na anatomia foliar de quatro espécies de grama. Planta Daninha, 28(3), 551-60.

Delgado, A. M. R., Hipolito, H. C., Prado, R., Castro, M. D. L. \& Franco, A. R. (2012). Limited uptake, translocation and enhanced metabolic degradation contribute to glyphosate tolerance in Mucuna pruriens var. utilis plants. Phytochemistry, 73, 34-41.

Duke, S. O. \& Powles, S. B. (2008). Glyphosate: a once-in-a-century herbicide. Pest Management Science, 64(4), 319-25.

Duke, S. O. The history and current status of glyphosate. (2018). Pest Management Science, 74(5), 1027-34.

Ferreira, E. A., Procópio, S. O., Silva, E. A. M., Silva, A. A. \& Rufino, R. J. N. (2003). Estudos anatômicos de folhas de espécies de plantas daninhas de grande ocorrência no Brasil: IV-Amaranthus deflexus, Amaranthus spinosus, Alternanthera tenella e Euphorbia heterophylla. Planta Daninha, 21(2), 263-71.

Giraldeli, A. L., San Gregorio, J. P. R., Monquero, P. A., Aguillera, M. M. \& Ribeiro, N. M. (2017). Weeds hosts of nematodes in sugarcane culture. Planta Daninha, 35.e017156815.

Hamilton, R. J. Waxes: chemistry, molecular biology and functions. Orly Press, 1995. 149 p.

McAllister, R. S. \& Haderlie, L. C. (1985). Translocation of ${ }^{14} \mathrm{C}$-Glyphosate and ${ }^{14} \mathrm{CO}_{2}$-Labeled Photoassimilates in Canada Thistle (Cirsium arvense). Weed Science, 33, 153-59.

Melo, E. B., da Silva, A. A. P., Oliveira Neto, A. M., Guerra, N., Vilar, C. C., Bottega, E. L. \& Goes Maciel, C. D. (2013). Alternativas para a catação química de touceiras de capim-colonião e capim-braquiária em cana-soca. Revista Brasileira de Herbicidas, 12(3), $307-17$.

Monquero, P. A., Christoffoleti, P. J., Osuna, M. D. \& De Prado, R. A. (2004). Absorção, translocação e metabolismo do glyphosate por plantas tolerantes e suscetíveis a este herbicida. Planta Daninha, 22(3), 445-51.

Monquero, P. A., Penha, A. S., Orzari, I. \& Hirata, A. C. S. (2011). Seletividade de herbicidas em mudas das espécies nativas Acacia polyphylla, Enterolobium contortisiliquum (Fabaceae), Ceiba speciosa e Luehea divaricata (Malvaceae). Planta Daninha, 29(1), 159-68.

Oliveira Neto, A. M., de Goes Maciel, C. D., Guerra, N., de Ramos Lima, G. G. \& Júnior, L. C. S. (2011). Manejo químico de adubos verdes para sucessão da cana-de-açúcar em sistema de cultivo mínimo. Revista Brasileira de Herbicidas, 10(2), 86-94.

Silva, G. B. F., Azania, C. A. M., Novo, M. C. S. S., Wutke, E. B., Zera, F. S. \& Azania, A. A. P. M. (2012). Tolerância de espécies de mucuna a herbicidas utilizados na cultura da cana-de-açúcar. Planta Daninha, 30(3), 589-97. 
Research, Society and Development, v. 10, n. 4, e49710414355, 2021

(CC BY 4.0) | ISSN 2525-3409 | DOI: http://dx.doi.org/10.33448/rsd-v10i4.14355

Silva, G. B. F., Azania, C. A. M., Novo, M. C. S. S., Wutke, E. B., Zera, F. S. \& Azania, A. A. P. M. (2013). Superação da profundidade de semeadura e densidades de palha para Mucuna aterrima, Mucuna deeringiana e Mucuna cinerea. Planta Daninha, 31(2), 313-17, 2013.

Sociedade Brasileira da Ciência das Plantas Daninhas - SBCPD. Procedimentos para instalação, avaliação e análise de experimentos com herbicidas. Londrina: SBCPD, 1995. 42 p.

Souza, G. S. F., Martins, D., Pereira, M. R. R. \& Bagatta, M. V. B. (2014). Action of rain on the efficiency of herbicides applied post-emergence in the control of Senna obtusifolia. Revista Ciência Agronômica, 45(3), 550-57.

Taiz, L. \& Zeiger, E. Fisiologia vegetal, 5 ed. Porto Alegre: Artmed, 2013. 918p.

Trezzi, M. M., Cruz, R. A., Rojano-Delgado, A. M., Alcántara, E., Pagnoncelli Jr., F. B., Viecelli, M., Diesel, F., Pacheco, V. \& Prado, R. (2021). Influence of temperature on the retention, absorption and translocation of fomesafen and imazamox in Euphorbia heterophylla. Pesticide Biochemistry and Physiology, 104794.

Velini, E., Duke, S. O., Trindade, M. L. B., Meschede, D. K. \& Carbonari, C. A. Modo de ação do glyphosate. In: Velini, E. D.; Meschede, D. K.; Carbonari, C. A.; \& Trindade, M. L. B. editores. Glyphosate. Botucatu: FEPAF; 2009. p. 113-33.

Zanatta, J. F., Procópio, S. D. O., Manica, R., Pauletto, E. A., Cargnelutti Filho, A., Vargas, L., Sganzerla, D. C., Rosenthal, M. D. A. \& Pinto, J. J. O. (2007). Teores de água no solo e eficácia do herbicida glyphosate no controle de Euphorbia heterophylla. Planta Daninha, 25(4), 799-811.

Wutke, E. B. Adubação verde: manejo da fitomassa e espécies utilizadas no Estado de São Paulo. In: Wutke, E. B., Bulisani, E. A. \& Mascarenhas, H. A. A. (Ed.). Curso sobre adubação verde no Instituto Agronômico. Campinas: Instituto Agronômico, 1993. p.17-29. (Documentos, 35). 